\title{
Capacity Analysis of an IEEE 802.11n System in a Residential House Based on Estimated Specular and Dense Multipath Components
}

\author{
E. Tanghe ${ }^{1, *}$, D. P. Gaillot ${ }^{2}$, D. Plets ${ }^{1}$, W. Joseph ${ }^{1}$, M. Liénard ${ }^{2}$, W. De Ketelaere ${ }^{3}$, L. Martens ${ }^{1}$ \\ ${ }^{1}$ iMinds-INTEC/WAVES, Ghent University, Technologiepark-Zwijnaarde 15, Ghent, Belgium \\ ${ }^{2}$ IEMN/TELICE, University of Lille 1, Bâtiment P3, Villeneuve d'Ascq, France \\ ${ }^{3}$ Excentis, Gildestraat 8, Ghent, Belgium \\ *emmeric.tanghe@intec.ugent.be
}

\begin{abstract}
This paper analyses the Multiple-Input Multiple-Output (MIMO) capacity in a residential house. The capacity calculation is based on physical radio channels estimated from channel sounding measurements. Both specular and dense multipath components are estimated from the channel sounding data with the RiMAX maximum-likelihood algorithm. In particular, the capacity analysis is made for a $2 \times 2$ IEEE 802.11n MIMO system that conforms to the legacy mode of the standard and operates in the $2.4 \mathrm{GHz}$ ISM band. The contribution of dense multipath to the channel capacity is quantified by calculating the capacity of radio channels for which the dense multipath is ignored and only the specular multipath is retained. It is found that ignoring dense multipath underestimates the capacity on average by $1.1,9.1$, and $37.8 \%$ for line-of-sight, obstructed line-of-sight, and non-line-of-sight situations, respectively.
\end{abstract}

\section{Introduction}

In this paper, the wireless capacity of an IEEE $802.11 \mathrm{n}$ system in a residential house is simulated starting from measurement-based multipath radio channels. The IEEE $802.11 \mathrm{n}$ system operates in the $2.4 \mathrm{GHz}$ frequency band. A capacity simulation methodology is developed wherein the physical-layer parameters of IEEE 802.11n (FFT size, guard interval, etc.) can be configured manually. The geometrical properties of the MIMO antenna arrays can also be specified, together with the full-polarimetric complex antenna radiation patterns. The proposed capacity-estimation methodology is useful for the inclusion in indoor network planning software such as [1].

The multipath radio channels are extracted from frequency-swept radio channel sounding measurements done with a vector network analyzer and virtual antenna arrays. The multipath components are estimated from the measurement data with the RiMAX maximum-likelihood method [2]. The multipath channels consist of both Specular Multipath Components (SMCs) and Dense Multipath Components (DMC). The inclusion of DMC in radio channel models has been promoted by the recent European COST actions 2100 [3] and IC1004 [4]. DMC follows from the observation that SMCs alone often appear to be insufficient to account for all multipath energy in a radio channel. Capacity analyses of DMC-inclusive radio channels are scarce in literature: examples can be found in [5] and [6], both for outdoor environments. 


\section{Channel sounding measurements}

Frequency-swept channel sounding measurements were done in different rooms of detached residential house located in a rural area. A Vector Network Analyzer (VNA, Rohde \& Schwarz ZNB20) was used to sample the radio channel between $2.47 \mathrm{GHz}$ and $2.55 \mathrm{GHz}(80 \mathrm{MHz}$ bandwidth). The channel is sampled in $M_{f}=200$ equally spaced frequency points. The VNA calibration includes the feeder cables for the transmitting antenna (Tx) and the receiving antenna (Rx).

At both the transmitting and the receiving side, an automatic positioning system was used to create virtual antenna arrays. At both link ends, the virtual array is made up of two verticallystacked planar horizontal Uniform Circular Arrays (UCAs) of eight antenna elements each. Thus, at the transmitting (receiving) side, the complete virtual array has $M_{T}=16\left(M_{R}=16\right)$ antennas. For each Tx and Rx position, one sweep the frequency range was taken. Dual-polarized patch antennas were used for the Tx and the Rx to register both the horizontal (H) and vertical (V) component of the electric field. The lowest position of the Tx and Rx patch antennas was $0.80 \mathrm{~m}$ above the floor. No people were present in the house during measurements because of the static radio channel requirement when using virtual antenna arrays.

The residential house has two floors of equal size. Fig. 1 shows a plan of the ground floor. In total, 16 Tx-Rx links were measured in and between different rooms in the house. For the first eight links, the Tx and Rx were positioned on the ground floor. The Tx was installed in the lowerleft corner in Fig. 1 (indicated as Tx1), while the Rx was moved between measurements to eight different locations across the different rooms on the ground floor (indicated as Rx1 through Rx8). For the last eight links, the Tx was moved to a fixed position on the first floor (Tx2), while the Rx was moved to the same eight positions on the ground floor as for the first eight links (Rx1 through $\mathrm{Rx} 8)$.

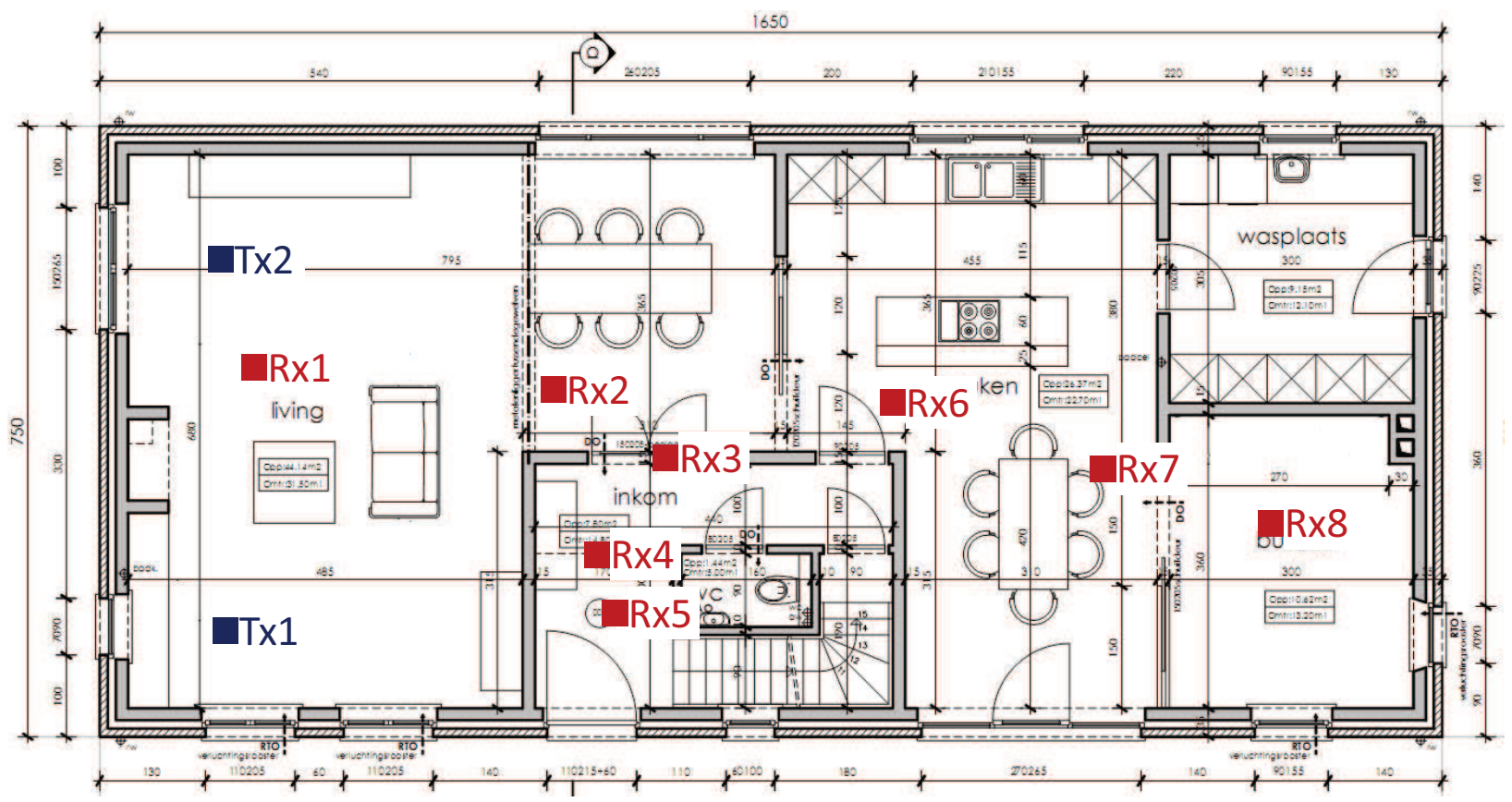

Figure 1. Floor plan + Tx and Rx locations 


\section{Specular and dense multipath estimation}

\subsection{Signal model for the complex channel gain}

It is assumed that the measured complex channel gain vectors $\boldsymbol{h}_{X Y} \in \mathbb{C}^{M_{R} M_{T} M_{f} \times 1}$ are made up of an SMC part $\boldsymbol{s}_{X Y}$, a DMC part $\boldsymbol{d}_{X Y}$, and a measurement noise part $\boldsymbol{n}_{X Y}$ :

$$
\boldsymbol{h}_{X Y}=\boldsymbol{s}_{X Y}\left(\boldsymbol{\theta}_{s, X Y}\right)+\boldsymbol{d}_{X Y}\left(\boldsymbol{\theta}_{d, X Y}\right)+\boldsymbol{n}_{X Y}\left(\sigma_{X Y}^{2}\right)
$$

The subscripts $X$ and $Y$ signify the polarization of the Tx and the Rx, respectively. $X$ and $Y$ are either horizontal $(H)$ or vertical $(V)$. It is well-established that $\boldsymbol{h}_{X Y}$ follows a complex Gaussian distribution [2]:

$$
\boldsymbol{h}_{X Y} \sim \mathcal{N}_{c}\left(\boldsymbol{s}_{X Y}\left(\boldsymbol{\theta}_{s, X Y}\right), \boldsymbol{R}_{X Y}\left(\boldsymbol{\theta}_{d, X Y}, \sigma_{X Y}^{2}\right)\right) .
$$

The vector $\boldsymbol{\theta}_{s, X Y}$ combines the multipath parameters describing the SMCs $\boldsymbol{s}_{X Y}$. Part of these multipath parameters are geometrical in nature: the angles of arrival and departure, and the times of arrival of the specular multipath components. The geometrical SMC parameters are identical across all four polarization subchannels $X Y$. Furthermore, $\boldsymbol{\theta}_{s, X Y}$ also includes the complex amplitudes $\gamma_{X Y}$ of the SMCs. The complex amplitudes are not the same between polarization subchannels because electromagnetic wave interactions depend on the polarization state of the incident wave.

The DMC $\boldsymbol{d}_{X Y}$ and the measurement noise $\boldsymbol{n}_{X Y}$ in (1) are characterized by the covariance matrix $\boldsymbol{R}_{X Y}$ in (2). This covariance matrix has the following structure [2]:

$$
\begin{aligned}
& \boldsymbol{R}_{X Y}\left(\boldsymbol{\theta}_{d, X Y}, \sigma_{X Y}^{2}\right) \\
& =\boldsymbol{R}_{d, X Y}\left(\boldsymbol{\theta}_{d, X Y}\right)+\sigma_{X Y}^{2} \boldsymbol{I}_{M} \\
& =\boldsymbol{I}_{M_{R}} \otimes \boldsymbol{I}_{M_{T}} \otimes \boldsymbol{R}_{f, X Y}\left(\boldsymbol{\theta}_{d, X Y}\right)+\sigma_{X Y}^{2} \boldsymbol{I}_{M} .
\end{aligned}
$$

In (3), $\boldsymbol{I}_{\kappa}$ is the identity matrix of size $\kappa$, the $\otimes$ operator is the Kronecker product, and $M=$ $M_{R} M_{T} M_{f}$. In (3), the measurement noise is assumed to be independent and identically distributed complex Gaussian noise with variance $\sigma_{X Y}^{2}$. The DMC covariance matrix $\boldsymbol{R}_{d, X Y}$ is uncorrelated in the spatial domains at the receiver $\left(\boldsymbol{I}_{M_{R}}\right)$ and at the transmitter $\left(\boldsymbol{I}_{M_{T}}\right)$, and is correlated in the frequency domain $\left(\boldsymbol{R}_{f, X Y}\right)$. Without going into detail, the mathematical expression of $\boldsymbol{R}_{f, X Y}$ is derived from the DMC power delay profile $\psi_{X Y}(\tau)$ as function of time-delay $\tau$. The DMC power delay profile follows an exponential law [2]:

$$
\psi_{X Y}(\tau)=\alpha_{d, X Y} e^{-\beta_{d, X Y}\left(\tau-\tau_{d, X Y}\right)} .
$$

In (4), $\alpha_{d, X Y}, \beta_{d, X Y}$, and $\tau_{d, X Y}$ are parameters describing the DMC of polarization subchannel $X Y$, which are combined in the DMC parameter vector $\boldsymbol{\theta}_{d, X Y}$.

\subsection{RiMAX maximum-likelihood estimation}

Maximum-likelihood estimates of $\hat{\boldsymbol{\theta}}_{s, X Y}, \hat{\boldsymbol{\theta}}_{d, X Y}$, and $\hat{\sigma}_{X Y}^{2}$ are obtained from the radio channel measurements using the RiMAX framework [2]. Our RiMAX code contains an implementation of the Effective Aperture Distribution Function (EADF) framework detailed in [7]. The EADF accounts for the effect that the Tx and $\mathrm{Rx}$ radiation patterns have on the channel sounding data (a so-called non-channel effect). 
The RiMAX algorithm is an iterative algorithm. In each iteration, the following procedure takes place. Firstly, the power angular-delay spectrum is calculated from the measured channel gain vector $\boldsymbol{h}_{X Y}$. From this spectrum, five SMCs are initialized as the five largest local maxima. These five SMCs serve as rough initial estimates of the corresponding true SMCs. Secondly, the rough SMC estimates are made to converge towards their true counterpart by solving the maximumlikelihood optimization problem with the fast-converging Levenberg-Marquardt algorithm. In the final step, the DMC and measurement noise (i.e., the measured channel minus the five SMCs) are fitted to the data models in (3) and (4).

At the start of each iteration, the five SMCs estimated in the previous iteration are removed from the measured channel gain vector to allow the algorithm to search for five new paths. Furthermore, the reliability of each estimated SMC is checked with a criterion based on the SMC power estimate and its estimation error variance (see [2] and [8] for further details). Estimated SMCs that fail this criterion are removed from further analysis. The reliability criterion is also used to create a stop condition for the RiMAX iterations. When all five SMCs in the same RiMAX iteration prove to be unreliable, then the radio channel is considered to be exhausted of reliable SMC and the RiMAX algorithm is stopped.

To give an example, Fig. 2 shows the estimated SMCs of the Tx1-Rx3 link. SMCs departing from the Tx and arriving at the Rx are shown as grey and black lines, respectively. The SMC line length equals the path length of the specular component scaled to the figure's dimensions. The thickness of the SMC lines is proportional to the specular path's power. For the Tx1-Rx3 link in Fig. 2, the RiMAX iterations stopped after the algorithm found $P=98$ SMCs. For all 16 Tx-Rx links, the number of SMCs $P$ varied from 11 to 155.

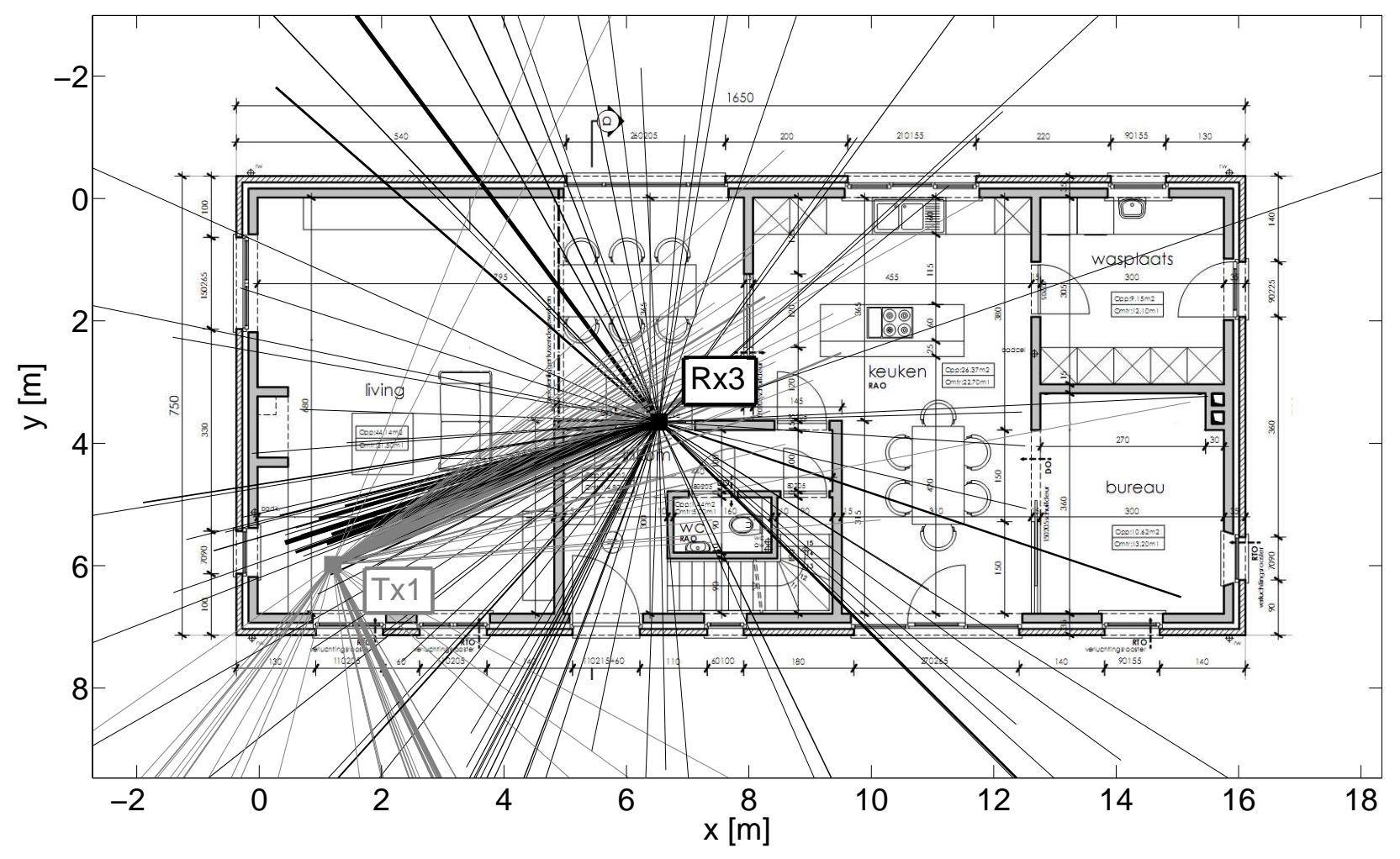

Figure 2. Estimated SMCs of the Tx1-Rx3 link 


\section{Simulated IEEE $802.11 \mathrm{n}$ legacy mode system}

The capacity simulation is performed for an access point-client link that implements the IEEE 802.11n wireless communication standard [9]. We choose channel 6 in the $2.4 \mathrm{GHz}$ ISM band for this link (center frequency is $2.437 \mathrm{GHz}$ and bandwidth is $20 \mathrm{MHz}$ ). The simulated system has two antennas at both the $\mathrm{Rx}$ and the Tx side $\left(M_{R}^{\prime}=M_{T}^{\prime}=2\right)$. The two-antenna arrays are positioned at the same Tx-Rx locations as in the measurement campaign (Fig. 1). The two antennas of both arrays are separated along the $\mathrm{x}$-axis in Fig. 2 by half a wavelength corresponding to the channel 6 center frequency. All four antennas are vertically polarized half-wave dipole antennas and have a maximum gain of $2.15 \mathrm{dBi}$.

IEEE 802.11n OFDM in legacy mode uses $20 \mathrm{MHz}$ wide channels and a 64-point FFT of which 52 points are datacarriers. The radio channel will be simulated only in the $M_{f}^{\prime}=52$ datacarriers as only the datacarriers contribute to the channel capacity. Furthermore, the capacity simulation takes the $800 \mathrm{~ns}$ guard interval used in IEEE 802.11n legacy mode into account. With this guard interval, the OFDM symbol duration $T_{s}$ amounts to $4 \mu \mathrm{s}$, corresponding to a symbol rate $f_{s}$ of 250 ksymbols/s.

\section{Capacity calculation}

\subsection{Radio channel generation}

Radio channel gain vectors ${ }^{1} \boldsymbol{h}^{\prime} \in \mathbb{C}^{M_{R}^{\prime} M_{T}^{\prime} M_{f}^{\prime} \times 1}$ are constructed in accordance with the IEEE $802.11 \mathrm{n}$ system specifications given in Section 4. The channel gain vectors are the sum of an SMC part $s^{\prime}$ and a DMC part $\boldsymbol{d}^{\prime}$ :

$$
h^{\prime}=s^{\prime}+d^{\prime}
$$

The SMC part $s^{\prime}$ is constructed as follows:

$$
\boldsymbol{s}^{\prime}=\sum_{\substack{X \in\{H, V\} \\ Y \in\{H, V\}}}\left[\left(\boldsymbol{G}_{R, Y}^{\prime} \circ \boldsymbol{A}_{R}^{\prime}\right) *\left(\boldsymbol{G}_{T, X}^{\prime} \circ \boldsymbol{A}_{T}^{\prime}\right) * \boldsymbol{A}_{f}^{\prime}\right] \hat{\gamma}_{X Y}
$$

In (6), the $\circ$ and $*$ operators signify the Schur-Hadamard and the column-wise Khatri-Rao matrix products, respectively. $\boldsymbol{A}_{R}^{\prime} \in \mathbb{C}^{M_{R}^{\prime} \times P}, \boldsymbol{A}_{T}^{\prime} \in \mathbb{C}^{M_{T}^{\prime} \times P}$, and $\boldsymbol{A}_{f}^{\prime} \in \mathbb{C}^{M_{f}^{\prime} \times P}$ are steering matrices of complex exponentials. $\boldsymbol{A}_{R}^{\prime}\left(\boldsymbol{A}_{T}^{\prime}\right)$ depends on the Rx (Tx) array's geometry and on the SMC angles of arrival (departure). The steering matrix $\boldsymbol{A}_{f}^{\prime}$ depends on the simulated frequency points and on the SMC times of arrival. Furthermore, $\boldsymbol{G}_{R, Y}^{\prime} \in \mathbb{C}^{M_{R}^{\prime} \times P}$ and $\boldsymbol{G}_{T, X}^{\prime} \in \mathbb{C}^{M_{T}^{\prime} \times P}$ are gain matrices that multiply the elements of the Rx and Tx steering matrices with the appropriate antenna directivity. Finally, $\hat{\gamma}_{X Y} \in \mathbb{C}^{P \times 1}$ is a vector of the estimated SMC complex amplitudes in polarization subchannel $X Y$. The sum in (6) is taken over all four polarization subchannels.

For the simulation of the DMC part $\boldsymbol{d}^{\prime}$ in (5), we first reconstruct the DMC covariance matrix $\boldsymbol{R}_{d, X Y}^{\prime} \in \mathbb{C}^{M^{\prime} \times M^{\prime}}$ based on (3) and the estimated DMC parameter vector $\hat{\boldsymbol{\theta}}_{d, X Y}$ :

$$
\boldsymbol{R}_{d, X Y}^{\prime}=\boldsymbol{I}_{M_{R}^{\prime}} \otimes \boldsymbol{I}_{M_{T}^{\prime}} \otimes \boldsymbol{R}_{f, X Y}^{\prime}\left(\hat{\boldsymbol{\theta}}_{d, X Y}\right)
$$

\footnotetext{
${ }^{1}$ In this section, the prime symbol is used to denote variables that follow from radio channel simulation. This is to distinguish them from the same variables without prime that are associated with the channel sounding and estimation procedures in Sections 2 and 3.
} 
Following, an eigendecomposition factorization is performed on the reconstructed DMC covariance matrix:

$$
\boldsymbol{R}_{d, X Y}^{\prime}=\boldsymbol{U}_{X Y} \boldsymbol{\Lambda}_{X Y} \boldsymbol{U}_{X Y}^{\dagger}
$$

In (8), $\dagger$ is the Hermitian transpose. The columns of $\boldsymbol{U}_{X Y}$ are made up of the eigenvectors of $\boldsymbol{R}_{d, X Y}^{\prime}$, and $\Lambda_{X Y}$ is a diagonal matrix of the real non-negative eigenvalues. The factorization in (8) is used to create observations of $\boldsymbol{d}^{\prime}$ :

$$
\boldsymbol{d}^{\prime}=\sum_{\substack{X \in\{H, V\} \\ Y \in\{H, V\}}}\left(\boldsymbol{U}_{X Y} \boldsymbol{\Lambda}_{X Y}^{1 / 2}\right) \boldsymbol{z}_{X Y}
$$

In (9), $\boldsymbol{z}_{X Y} \in \mathbb{C}^{M_{R}^{\prime} M_{T}^{\prime} M_{f}^{\prime} \times 1}$ contains independent and identically distributed complex Gaussian random variables with unit variance. For each Tx-Rx link, 200 independent samples of $\boldsymbol{h}^{\prime}$ are generated.

\subsection{Capacity of generated radio channels}

Per Tx-Rx link, the capacity is calculated for two types of simulated channel. The first channel is the simulated channel $\boldsymbol{h}^{\prime}$ in (5) which includes both the estimated SMC and DMC. For the second channel, we only consider the simulated SMC part $s^{\prime}$ in (6) while ignoring the DMC part $\boldsymbol{d}^{\prime}$ in (9). For the calculation of capacity, the simulated channel and SMC response vectors $\boldsymbol{h}^{\prime}$ and $\boldsymbol{s}^{\prime}$ are each first converted into $M_{f}^{\prime}$ channel and SMC matrices $\boldsymbol{H}_{k}^{\prime}$ and $\boldsymbol{S}_{k}^{\prime} \in \mathbb{C}^{M_{R}^{\prime} \times M_{T}^{\prime}}$, where $k$ denotes the OFDM subcarrier. The simulated capacities $C^{\prime}$ are then computed by summing the partial capacities of each individual OFDM subcarrier [10]:

$$
\begin{aligned}
C_{\mathrm{SMC}+\mathrm{DMC}}^{\prime}(\rho) & =\sum_{k=1}^{M_{f}^{\prime}} \mathbb{E}_{r}\left[f_{s} \log _{2}\left(\operatorname{det}\left(\boldsymbol{I}_{M_{R}^{\prime}}+\frac{\rho}{M_{T}^{\prime}} \alpha_{k} \boldsymbol{H}_{k}^{\prime}\left(\boldsymbol{H}_{k}^{\prime}\right)^{\dagger}\right)\right)\right] \\
C_{\mathrm{SMC}}^{\prime}(\rho) & =\sum_{k=1}^{M_{f}^{\prime}} f_{s} \log _{2}\left(\operatorname{det}\left(\boldsymbol{I}_{M_{R}^{\prime}}+\frac{\rho}{M_{T}^{\prime}} \alpha_{k} \boldsymbol{S}_{k}^{\prime}\left(\boldsymbol{S}_{k}^{\prime}\right)^{\dagger}\right)\right)
\end{aligned}
$$

In (10) and (11), $\rho$ is the Signal-to-Noise Ratio (SNR) at one of the receiving antennas, averaged over all OFDM subcarriers. The expectation $\mathbb{E}_{r}[\cdot]$ in (10) is taken over the 200 independent realizations of the simulated channel. In (11), no expectation is needed because the SMC are deterministic in nature and remain constant over the 200 realizations. Furthermore, $\alpha_{k}$ is a normalization factor chosen such that the expected value of each element of the complete (i.e., SMC and DMC inclusive) channel response vector $\boldsymbol{h}^{\prime}$ equals one:

$$
\alpha_{k}=\frac{M_{R}^{\prime} M_{T}^{\prime}}{M_{f}^{\prime} \sum_{m=1}^{M_{R}^{\prime}} \sum_{n=1}^{M_{T}^{\prime}} \mathbb{E}_{r}\left[\left|\boldsymbol{H}_{k}^{\prime}(m, n)\right|^{2}\right]}
$$

Fig. 3 shows the simulated IEEE 802.11n capacity versus SNR of the same Tx-Rx link as in Fig. 2. It is clear that excluding DMC from the calculation leads to an underestimation of the true capacity given by the SMC and DMC inclusive channel. It has previously been argued that DMC can contribute significantly to the channel capacity [5]. The stochastic randomness of the DMC 
gives rise to increased multipath diversity in the radio channel, effectively benefiting the achievable IEEE 802.11 capacity.

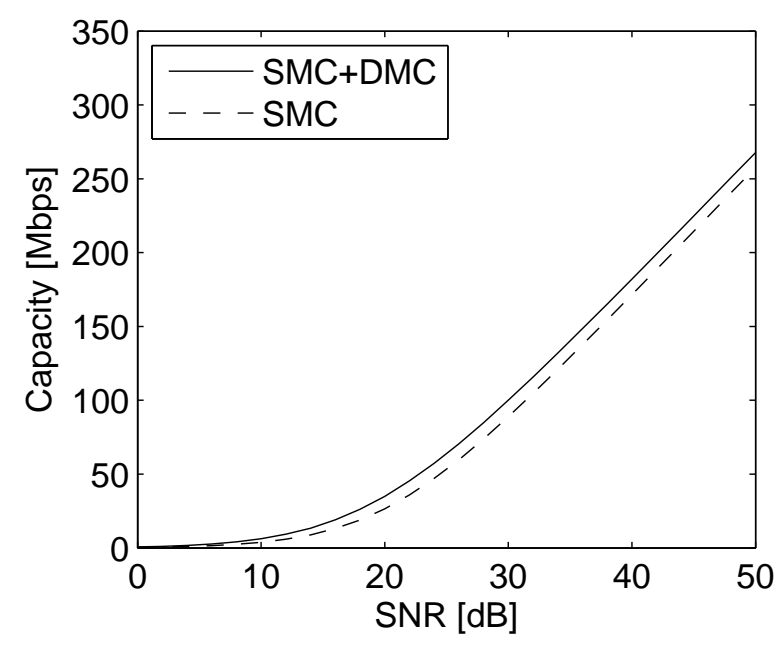

Figure 3. Capacity versus SNR for the Tx1-Rx3 link

We define the capacity underestimation error of SMC-only channels compared to SMC and DMC inclusive complete channels as the (percentual) relative capacity difference $\Delta C^{\prime}$ between both types of channel. It will be used in the next section to quantify the capacity underestimation of a realistic 802.11 n usage scenario.

$$
\Delta C^{\prime}(\rho)=100 \cdot \frac{C_{\mathrm{SMC}+\mathrm{DMC}}^{\prime}(\rho)-C_{\mathrm{SMC}}^{\prime}(\rho)}{C_{\mathrm{SMC}+\mathrm{DMC}}^{\prime}(\rho)}
$$

\section{Capacity of SMC-only versus SMC and DMC inclusive channels}

In this section, the capacity error $\Delta C^{\prime}$ of SMC-only channels is evaluated for a typical IEEE 802.11n usage scenario in a residential setting. A simple link budget calculation is performed for this scenario with the goal of obtaining a realistic estimate of the receiver Signal-to-Noise Ratio (SNR), called the application SNR $\rho_{A}$ in the following. The application SNR follows the SNR definition in (10) and (11), in the sense that it is the SNR at one of the receiving antennas averaged over all OFDM subcarriers. The application SNR in $\mathrm{dB}$ is calculated as:

$$
\rho_{A}=P+P G-N
$$

Herein,

$$
\begin{aligned}
P & =15 \mathrm{dBm}-10 \log _{10}\left(M_{T}^{\prime} M_{f}^{\prime}\right) \\
P G & =10 \log _{10}\left(\mathbb{E}_{m, k, r}\left[\left|\sum_{n=1}^{M_{T}^{\prime}} \boldsymbol{H}_{k}^{\prime}(m, n)\right|^{2}\right]\right) \\
N & =N_{0}+10 \log _{10}(B W)+N F
\end{aligned}
$$


$P$ is the transmitted power per transmitting antenna and per OFDM subcarrier. For this scenario, we choose a total RF input power of $15 \mathrm{dBm}$ which is distributed equally between the $M_{T}^{\prime}$ transmitting antennas and the $M_{f}^{\prime}$ subcarriers. $P G$ is the path gain in dB calculated from the simulated channel matrices $\boldsymbol{H}_{k}^{\prime}$. The expectation $\mathbb{E}_{m, k, r}[\cdot]$ in (16) is taken simultaneously over the receiving antennas, the subcarriers, and the channel realizations. The path gain includes the radiation patterns of the transmitting and receiving antennas from the manner in which the channel matrices are constructed in Section 5.1. $N$ is the noise power at one of the receiving antennas in $\mathrm{dBm}$, consisting of thermal noise and a noise figure. $N_{0}$ is the thermal noise spectral density at $25^{\circ} \mathrm{C}\left(N_{0}=-173.9 \mathrm{dBm} / \mathrm{Hz}\right)$ and $B W$ is the bandwidth of the IEEE $802.11 \mathrm{n}$ legacy system $(B W$ $=20 \mathrm{MHz}$ ). For the noise figure $N F$, a value of $10 \mathrm{~dB}$ is chosen.

Fig. 4 shows the capacities $C_{\mathrm{SMC}}^{\prime}\left(\rho_{A}\right)$ (white bar) and $C_{\mathrm{SMC}+\mathrm{DMC}}^{\prime}\left(\rho_{A}\right)$ (black bar) for each Tx$\mathrm{Rx}$ link. The application SNR $\rho_{A}$ in $\mathrm{dB}$ is given in brackets below the Rx numbers. The capacity error $\Delta C^{\prime}\left(\rho_{A}\right)$ is shown in red.
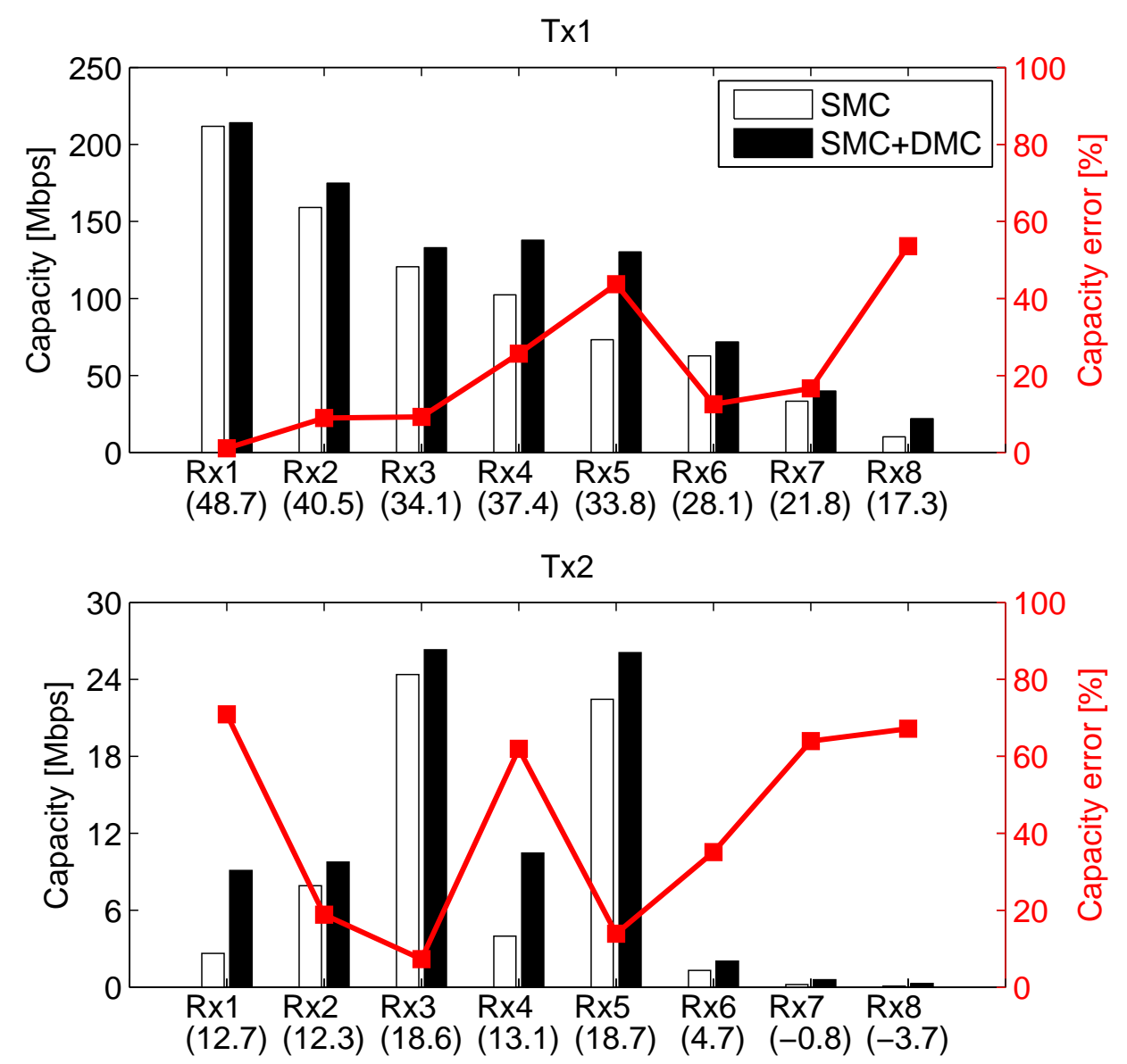

Figure 4. Usage scenario capacity for each Tx-Rx link + capacity error of SMC-only channels

Of all links, Tx1-Rx1 is the only link with a clear Line-of-Sight (LoS) component. The capacity error for Tx1-Rx1 amounts to $1.1 \%$ and is the lowest of all 16 links. The observation that the capacity error is nearly negligible for LoS is easily explained by the powerful LoS multipath component overshadowing the power of the DMC. As such, the capacity of Tx1-Rx1 is largely carried by the LoS component and the secondary SMC. We consider the shadowing conditions of the Tx1-Rx2 and Tx1-Rx3 links to be Obstructed Line-of-Sight (OLoS). We define OLoS as the 
situation wherein the LoS component is obstructed, but no through-wall propagation is needed to reach the Rx. The capacity error of the Tx1-Rx2 and Tx1-Rx3 links is 9.0 and $9.3 \%$, respectively (9.1\% on average). The shadowing conditions of the remaining $13 \mathrm{Tx}-\mathrm{Rx}$ links are categorized as Non-Line-of-Sight (NLoS). For the NLoS links, a significant portion of the multipath energy needs to travel through walls or the ground floor ceiling in order to reach the Rx. The capacity error of the NLoS links varies between 7.3 and $70.9 \%$ (37.8\% on average).

It is observed that the capacity error on average increases as the shadowing conditions become harsher. This is expected as increased shadowing means that a larger proportion of the radio channel power will be carried by the DMC. Shadowing of the SMC reduces their power to the point where the RiMAX estimator cannot reliably detect them among the scattered diffuse multipath [8]. In this case, these low-power SMC are automatically lumped together with the DMC, increasing the presence and the power of the latter. Finally, it is noteworthy that the capacity underestimation errors of SMC-only channels reported here are on par with those reported for a $4 \times 4$ MIMO system in an macro-cell outdoor environment in [7]. In [7], the capacity underestimation is equally close to $0 \%$ for LoS, and varies between 0 and about $73 \%$ for OLoS/NLoS.

\section{Conclusions}

This work presented a methodology to simulate the MIMO capacities of IEEE 802.11n links based on empirical multipath radio channels in a residential house. These radio channels include both specular and dense multipath components estimated with the RiMAX maximum-likelihood algorithm. The capacity calculations are done for a $2 \times 2$ MIMO system that employs the legacy mode of the IEEE $802.11 \mathrm{n}$ standard in the $2.4 \mathrm{GHz}$ band. It is found that ignoring dense multipath can underestimate the MIMO capacity significantly, except for line-of-sight situations. For our residential scenario, omitting dense multipath underestimates the capacity on average by 1.1, 9.1, and $37.8 \%$ for line-of-sight, obstructed line-of-sight, and non-line-of-sight situations, respectively.

\section{Acknowledgments}

The authors would like to thank Matthias Van den Bossche, M. Eng., for his help in building the channel sounding measurement system, and Jan De Beule, M. Sc. Eng., for his help during the measurements.

FORWARD is a project co-funded by iMinds, a digital research institute founded by the Flemish Government. Project partners are ArcelorMittal, Egemin, Excentis, Siemens, and Volvo, with project support from IWT. Emmeric Tanghe is a Post-Doctoral Fellow of the FWO-V (Research Foundation - Flanders, Belgium). This research was supported by the project IUAP BESTCOM, "BElgian network on STochastic modelling, analysis, design and optimization of COMmunication systems".

\section{References}

[1] D. Plets, W. Joseph, K. Vanhecke, E. Tanghe, and L. Martens, "Coverage Prediction and Optimization Algorithms for Indoor Environments," EURASIP Journal on Wireless Communications and Networking, vol. 1, pp. 1-23, 2012. 
[2] A. Richter, "Estimation of Radio Channel Parameters: Models and Algorithms," Ph.D. dissertation, Technische Universität Ilmenau, Fakültat für Elektrotechnik und Informationstechnik, Ilmenau, DE, 2005.

[3] C. Oestges, N. Czink, P. De Doncker, V. Degli-Esposti, K. Haneda, W. Joseph, M. Liénard, L. Liu, J. Molina-García-Pardo, M. Narandžić, J. Poutanen, F. Quitin, and E. Tanghe, Pervasive Mobile and Ambient Wireless Communications - COST Action 2100. Springer, 2012, ch. Radio Channel Modeling for 4G Networks, pp. 67-148.

[4] K. Haneda, W. Joseph, E. Tanghe, A. Bamba, U.-T. Virk, E.-M. Vitucci, C. Gustafson, J.-M. Molina-Garcia-Pardo, K. Witrisal, P. Kulakowski, P. Meissner, and E. Leitinger, Cooperative Radio Communications for Green Smart Environments - COST Action IC1004. River Publishers, 2016, ch. Indoor Wireless Communications and Applications, pp. 71-119.

[5] A. Richter, J. Salmi, and V. Koivunen, "Distributed Scattering in Radio Channels and its Contribution to MIMO Channel Capacity," in European Conference on Antennas and Propagation, Nice, FR, 2006, pp. 1-7.

[6] M. Landmann, M. Kaeske, R. Thoma, J.-I. Takada, and I. Ida, "Measurement Based Parametric Channel Modeling Considering Diffuse Scattering and Specular Components," in International Symposium on Antennas and Propagation, no. 1, 2007, pp. 153-156.

[7] M. Landmann, "Limitations of Experimental Channel Characterisation," Ph.D. dissertation, Technische Universität Ilmenau, Fakültat für Elektrotechnik und Informationstechnik, Ilmenau, DE, 2008.

[8] E. Tanghe, D. P. Gaillot, M. Liénard, L. Martens, and W. Joseph, "Experimental Analysis of Dense Multipath Components in an Industrial Environment," IEEE Transactions on Antennas and Propagation, vol. 62, no. 7, pp. 3797-3805, Jul. 2014.

[9] "IEEE Standard for information technology - Local and metropolitan area networks - Specific requirements - Part 11: Wireless LAN Medium Access Control (MAC) and Physical Layer (PHY) Specifications," IEEE Std 802.11n-2009, pp. 1-565, 2009.

[10] H. Bölcskei, CRC Handbook on Signal Processing for Mobile Communications. CRC Press, 2004, ch. Principles of MIMO-OFDM wireless systems, pp. 365-386. 